\title{
Effect of ordered array of magnetic dots on the dynamics of Josephson vortices in stacked SNS Josephson junctions under DC and $A C$ current
}

\author{
Golibjon R. Berdiyorov ${ }^{1}$, Sergey Savel'ev²,a, Feodor V. Kusmartsev², and François M. Peeters ${ }^{3}$ \\ 1 Qatar Environment and Energy Research Institute (QEERI), HBKU, Qatar Foundation, P.O. Box 5825, Doha, Qatar \\ 2 Department of Physics, Loughborough University, Leicestershire, LE11 3TU, UK \\ 3 Departement Fysica, Universiteit Antwerpen, Groenenborgerlaan 171, 2020 Antwerpen, Belgium
}

Received 3 August 2015 / Received in final form 10 September 2015

Published online 3 November 2015

(C) The Author(s) 2015. This article is published with open access at Springerlink.com

\begin{abstract}
We use the anisotropic time-dependent Ginzburg-Landau theory to investigate the effect of a square array of out-of-plane magnetic dots on the dynamics of Josephson vortices (fluxons) in artificial stacks of superconducting-normal-superconducting (SNS) Josephson junctions in the presence of external $\mathrm{DC}$ and AC currents. Periodic pinning due to the magnetic dots distorts the triangular lattice of fluxons and results in the appearance of commensurability features in the current-voltage characteristics of the system. For the larger values of the magnetization, additional peaks appear in the voltage-time characteristics of the system due to the creation and annihilation of vortex-antivortex pairs. Peculiar changes in the response of the system to the applied current is found resulting in a "superradiant" vortex-flow state at large current values, where a rectangular lattice of moving vortices is formed. Synchronizing the motion of fluxons by adding a small ac component to the biasing dc current is realized. However, we found that synchronization becomes difficult for large magnetization of the dots due to the formation of vortex-antivortex pairs.
\end{abstract}

\section{Introduction}

In recent years, considerable efforts have been devoted to the study of the dynamics of Josephson vortices (fluxons) in stacks of Josephson junctions (JJs) [1-13]. This is, e.g., motivated by its potential to use such Josephson systems for creating high-frequency electromagnetic oscillations (see Refs. $[14,15]$ for review). However, to increase the intensity of such sub-terahertz electromagnetic radiation sources, Josephson oscillations have to be in phase in the different junctions. This is because the emitted power is proportional to the square of the number of layers in the stack [16]. In inductively coupled JJs, the formation of the triangular Josephson flux lattice becomes energetically more favorable, resulting in decoherence (see, e.g., Ref. [17]). Therefore, the synchronization of Josephson oscillations in all the junctions becomes a major challenge in order to realize superradiance $[9,16]$. It has already been shown that synchronized oscillations can be obtained by a shunted circuit (see e.g., Ref. [18] and references therein) or by radiation fields [19-23]. It was predicted theoretically that the "superradiant" condition can be achieved without changing the external parameters or modifying the parameters of the junction itself. This is realized by using the transition from a triangular lattice to a more stable rectangular arrangement of moving fluxons which happens

\footnotetext{
a e-mail: s.saveliev@lboro.ac.uk
}

at some threshold fluxon velocity [24,25]. The stability range of the rectangular array of the vortices can be further increased by introducing an ordered array of pinning centers $[26,27]$. The pinning can be created by structural defects, which change the Josephson tunneling distance locally [12], or, alternatively, by forming pancake vortices at inclined magnetic fields [28]. The latter serves as magnetic pinning centers [29-31].

Here, we use the time-dependent Ginzburg-Landau theory to study the effect of magnetic pinning centers, created by a rectangular array of out-of-plane magnetic dots, on the dynamics of Josephson vortices in artificial stacks of superconducting-normal-superconducting (SNS) JJs. The motivation for such a study is manyfold. For example, the magnetic dot arrays can effectively be used as pinning centers for superconducting vortices in order to reduce the dissipation in the system [32]. For larger dot magnetization values, vortex-antivortex pairs are created under the dots [33-35], which can also be used for different applications, e.g., for spintronics devices. We found that the critical current for the transition from out-of-phase motion of vortices to an in-phase rectangular lattice decreases and, consequently the range of stability of this superradiant state increases due to the rectangular array of pinning centers. For larger dot magnetization, fluxonantifluxon pairs are created under the dots, which result in qualitative changes in the current-voltage $(I-V)$ characteristics of the system. We also studied the dynamic response 
of the system to an applied external AC current with the aim to increase the synchronization of the vortices. As a results of this work, interesting transport phenomena were discovered.

\section{Theoretical approach}

Our model system consists of a thin $(t \ll \xi(T), \lambda(T))$ superconducting film (of length $L$ and width $w$ ) containing an array of metallic Josephson junctions (i.e., weak links of width $\delta$ and period $d$ ). The superconducting transition temperature of the weak links is smaller than the one for the superconducting layers. We consider the case, where the weak link material is in the normal state. Such layered structures can be created using conventional superconducting materials (see, e.g., Ref. [36]). An array (with periods $d$ and $b$ in $x$ - and $y$-directions, respectively) of square magnetic dots (size $a$ and thickness $0.25 \xi$ ) is placed on top of the system as shown in Figure 1. An oxide layer (of thickness $0.25 \xi$ ) separates the magnetic dots from the superconductor and therefore we did not take into account the proximity effect at the magnetic dot/superconductor interface. The external current is applied through the metallic contacts in the presence of a uniform magnetic field. The following time-dependent Ginzburg-Landau (GL) equations are solved numerically for the considered system $[37,38]$ :

$$
\begin{array}{r}
\frac{u}{\sqrt{1+\gamma^{2}|\psi|^{2}}}\left(\frac{\partial}{\partial t}+i \varphi+\frac{\gamma^{2}}{2} \frac{\partial|\psi|^{2}}{\partial t}\right) \psi=(\nabla-i \mathbf{A})^{2} \psi \\
+\left(f(r)-|\psi|^{2}\right) \psi
\end{array}
$$

where $\psi$ is the superconducting order parameter, $\mathbf{A}$ is the vector potential and $\varphi$ is the electrostatic potential. The parameter $\gamma=2 \tau_{E} \psi_{0} / \hbar$ characterizes the chosen superconducting material (with $\tau_{E}$ being the inelastic electroncollision time and $\psi_{0}$ as the value of the order parameter at zero temperature and no applied field) and the coefficient $u$ governs the relaxation of the order parameter (i.e., the ratio between relaxation times for the phase and the amplitude of $\psi$ ). In this equation the critical temperature $T_{c}$ variations are accounted by the spatiallydependent parameter $f(r)$, which equals 1 inside the superconductor and less than 1 in the metallic regions. The definition of $f(r)$ follows from the anisotropic expansion coefficient of the Gibbs free-energy functional $\alpha=\alpha_{0} f(r)$ (see Ref. [39]). Equation (1) is coupled with the equation for the electrostatic potential $\varphi$ :

$$
\Delta \varphi=\operatorname{div}\left(\mathbf{j}_{\mathbf{s}}\right),
$$

where the superconducting current $\left(\mathbf{j}_{\mathbf{s}}\right)$ is given by:

$$
\mathbf{j}_{\mathbf{s}}=\left[\left(\psi^{*} \Delta \psi-\psi \Delta \psi^{*}\right)-\mathbf{A}|\psi|^{2}\right] / 2 i .
$$

This is nothing more than the condition for the conservation of the total current in the system. This approach has already been shown to be an effective tool in describing the properties of superconducting films with weak link

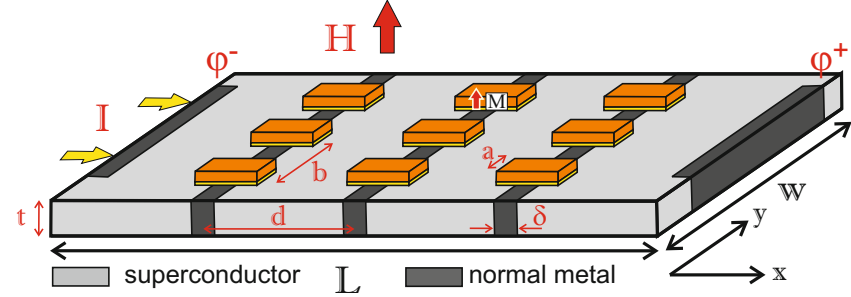

Fig. 1. Model system: a superconducting film (of thickness $t \ll \xi(T), \lambda(T)$ and lateral sizes $L$ and $w)$ with metallic Josephson junctions (i.e., weak links of width $\delta$ and period $d$ ) and with an array of antidots (size $a$ and period $b$ ) in the presence of a perpendicular magnetic field $(H)$ and external current $I$ applied through metallic contacts along the $x$-direction. $\varphi^{-}$and $\varphi^{+}$indicate the position of the voltage leads and a small arrow near $M$ indicates the direction of the magnetization of the dots.

arrays [39-42], as well as superconducting/metal multilayers [43]. Here, we express the length in units of the coherence length $\xi$, the order parameter $\psi$ in units of $\psi_{0}$, the vector potential $\mathbf{A}$ in units of $\Phi_{0} /(2 \pi \xi)$ (where $\Phi_{0}$ is the magnetic flux quantum) and the magnetic field in units of $H_{c 2}$. Time is scaled to the GL relaxation time $t_{0}=4 \pi \lambda^{2} / c^{2} \rho_{n}$ ( $\rho_{n}$ is the normal-state resistivity), the electrostatic potential (as well as the output voltage) is given in units of $V_{0}=c \Phi_{0} \rho_{n} / 8 \pi^{2} \lambda \xi$. The current density follows the relation $j_{0}=c \Phi_{0} / 8 \pi^{2} \lambda^{2} \xi$. The values for the material parameters $u$ and $\gamma$ are taken as $u=5.79$ and $\gamma=10$, which are found within the microscopic BCS theory for superconductors with weak depairing $[37,38]$. Further, we took the normal state resistivity as $\rho=18.7 \mu \Omega \mathrm{cm}$, the zero temperature coherence length was chosen as $\xi(0)=10 \mathrm{~nm}$ and the penetration depth was set as $\lambda(0)=200 \mathrm{~nm}$, all these numbers are characteristic of $\mathrm{Nb}$ thin films [44], from which we obtained $\tau_{G L} \approx 6.72 \mathrm{ps}$ and $V_{0} \approx 50 \mu \mathrm{V}$.

Assuming that the lateral dimensions of our sample are smaller than the Pearl length $\Lambda=2 \lambda^{2} / t$, we neglect the demagnetization effects and account for the uniform applied magnetic field using the gauge $\mathbf{A}=(-H y / 2, H x / 2)$. This reduces the problem to two dimensions. The vector potential of the stray magnetic field of the magnetic dots is calculated numerically and added to the vector potential of the uniform magnetic field in the superconducting plane. The procedure of calculating the vector potential of the magnetic field is as follows. First, we discretize the 3D magnet into $N_{x} \times N_{y} \times N_{z}$ cubes with moment $m=M * d V$, where $M$ is the magnetization of the material (in units of $\left.M_{0}=H_{c 2} / 4 \pi\right)$ and $d V$ is the volume of the elemental magnet. Then the vector potential in the point of interest becomes a sum of the vector potentials of all those dipoles (i.e. integral over the volume of the magnet). Vector potential of the point dipole is given by:

$$
\mathbf{A}=\frac{m}{a} \frac{\mathbf{r} \times x}{\rho^{2}}\left[\frac{x-a / 2}{\sqrt{\rho^{2}+(x-a / 2)^{2}}}-\frac{x+a / 2}{\sqrt{\rho^{2}+(x+a / 2)^{2}}}\right],
$$


where $a$ is the length of the dipole, $m$ is the dipole momentum and $\rho^{2}=y^{2}+z^{2}$. In our simulations we use $N_{x}=N_{y}=N_{z}=100$.

We apply a finite-difference representation of the order parameter, vector and scalar potentials on a uniform Cartesian space grid $(x, y)$, with typical grid spacing smaller than $0.2 \xi$ and use the link variable approach for the discretization of the equations [45]. We solve the coupled nonlinear differential equations self-consistently using the Euler iterative method (for $\psi$ ) and the Fourier transform technique (for $\varphi$ ). We use the Neumann boundary condition at all sample boundaries, except at the normal contacts where we use $\psi=0$ and $\left.\nabla \varphi\right|_{n}=-j$ boundary conditions. The applied current density $j$ is injected into the system in the form of normal current, which then converts to superconducting current over a distance of less than a few coherence lengths [46].

\section{DC biasing}

\subsection{Pinning free sample}

As a reference, we first study a system without magnetic dots in the presence of a uniform perpendicular magnetic field. The sample sizes are $L=42.5 \xi$ and $w=42.5 \xi$, and it consists of 5 Josephson junctions (i.e., weak link) of width $\delta=1.5 \xi$. The anisotropy coefficient is taken $f_{0}=0$. For the given system, we have constructed time-averaged voltage versus applied current $(I-V)$ characteristics for different values of an applied magnetic field. To avoid finite size effects, which are important for both type-I and typeII superconductors $[47,48]$, the voltage is measured at a distance of $10 \xi$ away from the normal contacts (see panel 1 in Fig. 2). Since the vector potential does not change with time, the voltage is determined as the difference in the scalar potential $\Delta \varphi$. We show in the main panel of Figure 2 the $I-V$ curves of the sample for different values of an external magnetic field. At zero magnetic field (solid-black curve), the resistive state transition occurs at $j_{c 1}=0.21 j_{0}$, below which the superconducting state is maintained. This state is characterized by periodic creation and annihilation of vortex antivortex pairs as was reported before for similar systems (see, e.g., Ref. [49]). The critical current for the resistive state transition decreases considerably with applied external magnetic field (dashed-red and dotted blue curves). In the presence of a magnetic field, a triangular arrangement of the moving Abrikosov vortex lattice is obtained (see panel 1). The triangular vortex lattice gets distorted at larger currents (panel 2), and finally a structural transition from triangular to a rectangular lattice occurs (panel 3). This final state is called the superradiant state $[17,24,25]$. Note that the current distorts the Josephson vortices, which then resemble the structure of fast-moving (kinematic) vortices in uniform superconducting films [50-52]. The superconducting-normal state transition current (the last jump in the $I-V$ curves) also decreases with increasing magnetic field.

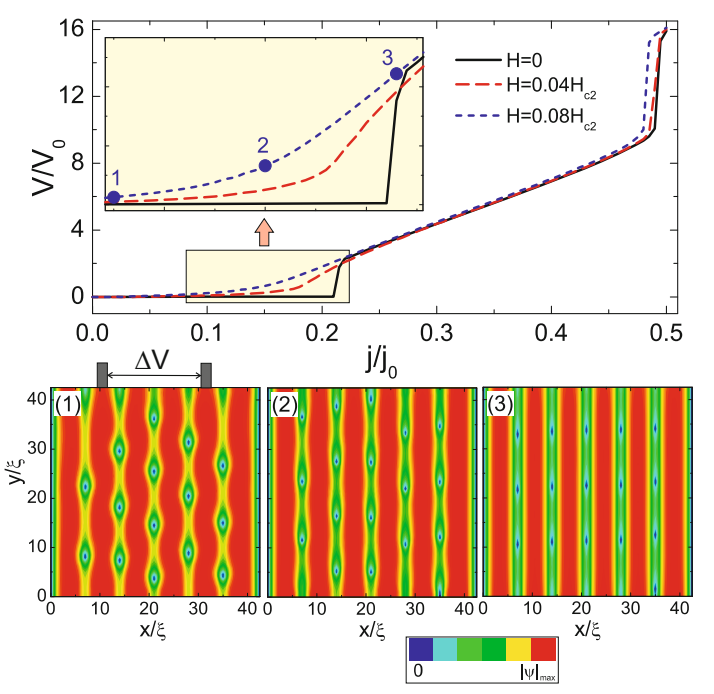

Fig. 2. Time averaged voltage vs. current density $(I-V)$ characteristics of the sample with 5 Josephson junctions and without magnetic dots for different values of the applied magnetic field $H$. The length of the sample is $L=42.5 \xi$, the width is $w=42.5 \xi$ and the width of the weak link layers is $\delta=1.5 \xi$. Panels 1-3 show snapshots of $|\psi|$ for current and field values indicated on the $I-V$ curves. Voltage probes are indicated in panel 1 by gray pillars. The anisotropy parameter is $f_{0}=0$.

\subsection{Effect of a lattice of magnetic dots}

Next, we introduce a lattice of magnetic dots and study its effect on the dynamics of the fluxons. As an example, we consider a square array of magnetic dots of size $a=2.5 \xi$ with period $b=8.5 \xi$ and separated by an oxide layer of $0.25 \xi$ from the superconductor. The size of the sample is the same as the one in Figure 2. Figure 3 shows the $I-V$ characteristics of the system for different values of an applied magnetic field. Results are shown for magnetization of the magnetic dots equal to $M=0.5 M_{0}$ (a) and $M=M_{0}(\mathrm{~b})$. The main effect of the magnetic dots is the reduction of the resistive state transition current. This is due to the suppression of the superconducting condensate under the magnetic dots. This creates a path for vortex motion. The resistive state at zero field is still characterized by periodic nucleation and annihilation of the Josephson fluxon-antifluxon pairs. At finite magnetic fields, a triangular lattice of moving fluxons are observed in the low resistive region of the $I-V$ graphs (point 1 and panel 1 of Fig. 3). As for the case of pinning-free sample (see Fig. 2), the in-phase oscillations of all the junctions in the stack are observed at higher currents (see panel 3 in Fig. 3). Such a stable superradiant flux-flow state is found in over wide region of the $I-V$ curve. When the magnetization of the dots is further increased (Fig. 3b), the jumps in the $I-V$ curves during the resistive state transition become less pronounced. The resistive state is now characterized by the nucleation (near magnetic dot edge) and annihilation of vortex-antivortex pairs (see panel 3 in Fig. 3b). The matching features in the $I-V$ curves becomes more pronounced for larger magnetization of the dots (dottedblue and dash-dotted-green curves in Fig. 3b). 


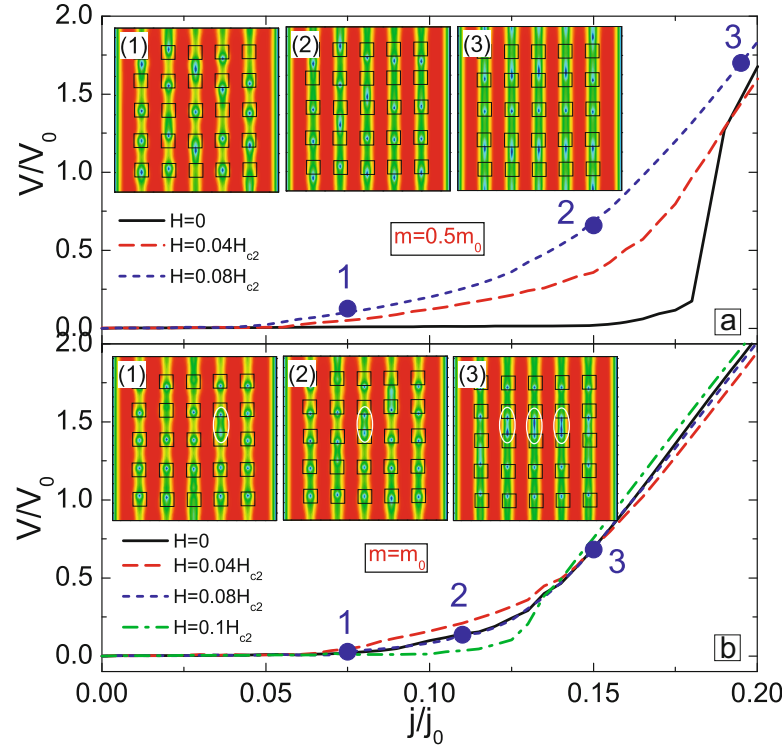

Fig. 3. $I-V$ curves of the sample with the same dimensions as in Figure 2 for different values of the applied magnetic field in the presence of magnetic dots with magnetization $M=0.5 M_{0}$ (a) and $M=M_{0}$ (b). The size of the magnetic dots is $a=$ $2.5 \xi$, the period is $b=8.5 \xi$ and they are separated from the superconductor by an insulating layer of thickness $0.25 \xi$. Insets show snapshots of $|\psi|$ for current and field values indicated on the $I-V$ curves. The location of the magnetic dots are shown by black squares and the white ellipses highlight the creation of vortex-antivortex pairs.

To analyze the resistive state properties of the system, and the effect of the magnetic dots on the structure of moving fluxons, we plot in Figure 4 the time evolution of the output voltage for the sample of Figure 3, together with the evolution of the vortex state, for an applied magnetic field $H=0.08 H_{c 2}$, current density $j=0.08 j_{0}$ and for magnetization $M=0.5 M_{0}$ (Fig. 4a) and $M=M_{0}$ (Fig. 4b). At this value of an external current for smaller dot magnetization, the output voltage oscillates periodically in time with several maxima and local minima in each period (Fig. 4a). The global minimum corresponds to the state where vortices are pinned by the dots (not shown here). Similarly, as in the case of a pinning-free sample, voltage peaks are observed during vortex penetration (inset 1 in Fig. 4a) and exit (inset 2 in Fig. 4a). The "hopping" of fluxons from one pinning center to the other results in faint kinks of the voltage curves. The triangular lattice of the fluxons are preserved for this particular value of applied current. The dynamics of Josephson vortices changes considerably for larger magnetization of the dots (Fig. 4b): the vortices propagate through the system by creation and annihilation of vortex-antivortex pairs near the dots (insets 1 and 2 in Fig. 4b). Each such event creates an extra peak in the $V(t)$ curves. However, some periodicity in the voltage curve is still visible.

We show in Figure 5 the $V(t)$ curves of the samples in Figure 4 for a larger value of an applied current $j=0.16 j_{0}$. For this value of the applied current, the superradiant state is formed, which is characterized by an in-phase

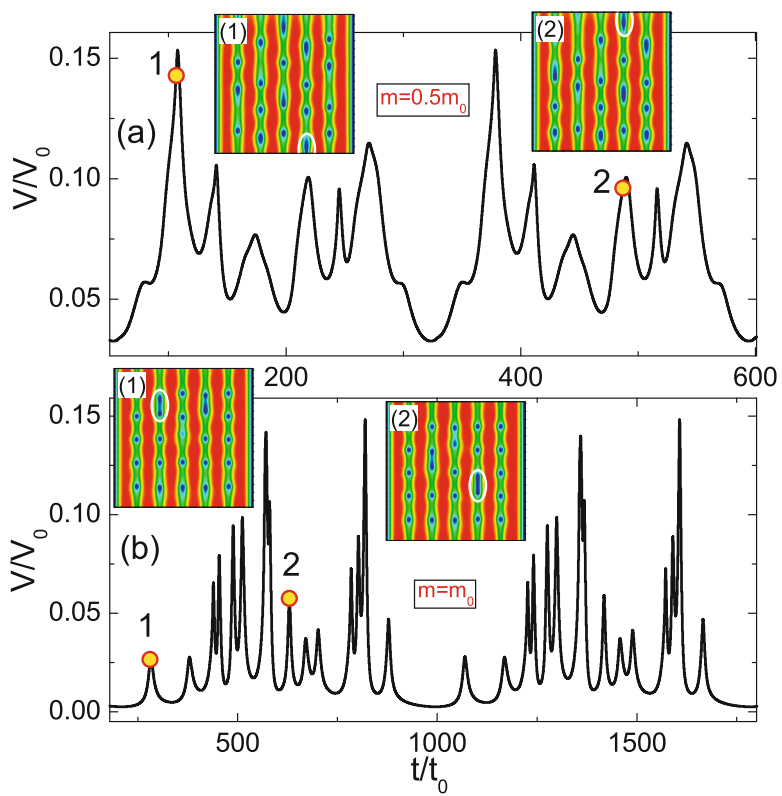

Fig. 4. Voltage vs. time $(V(t))$ characteristics of the sample of Figure 3 for $H=0.08 H_{c 2}, j=0.08 j_{0}$ and for magnetization $M=0.5 M_{0}$ (a) and $M=M_{0}$ (b). Insets show snapshots of $|\psi|$ at the times indicated by circles on the $V(t)$ curves. White ellipses highlight the penetration of vortices (a) and the annihilation of vortex-antivortex pairs near the edge of the magnetic $\operatorname{dots}(b)$.

motion of rectangularly arranged fluxons. Periodic oscillations are obtained in the $V(t)$ curves for both values of dot magnetization. For smaller magnetization, every voltage peak corresponds to the hopping of vortices from one dot to its neighbour or due to their interactions with the sample surface (see insets in Fig. 5a). Vortex-antivortex pairs are created near the magnetic dots for larger magnetizations, resulting in local maxima in the $V(t)$ curves (insets in Fig. 5b). The voltage signal amplitude is proportional to the number of vortex columns, moving between the pinning centers. Thus, as in the case of a pinning-free sample, we can identify two states for the moving vortex structure: a synchronous motion, where all the vortices jump from one magnetic dot to another at the same time, and an out-of-phase motion with a shift in the phase of vortices in neighboring layers.

\section{AC biasing}

As was demonstrated in the previous section, the superradiant state is only observed at larger values of external current and at smaller currents the resistive state is dominated by a non-rectangular array of moving vortices. This occurs despite the presence of rectangular pinning centers created by the magnetic dots. In this section, we study the dynamics of the Josephson vortices in the presence of both a time-periodic (and spatially uniform) AC current and a constant biasing DC current (also spatially uniform). The idea is to achieve in-phase oscillations of all the junctions 

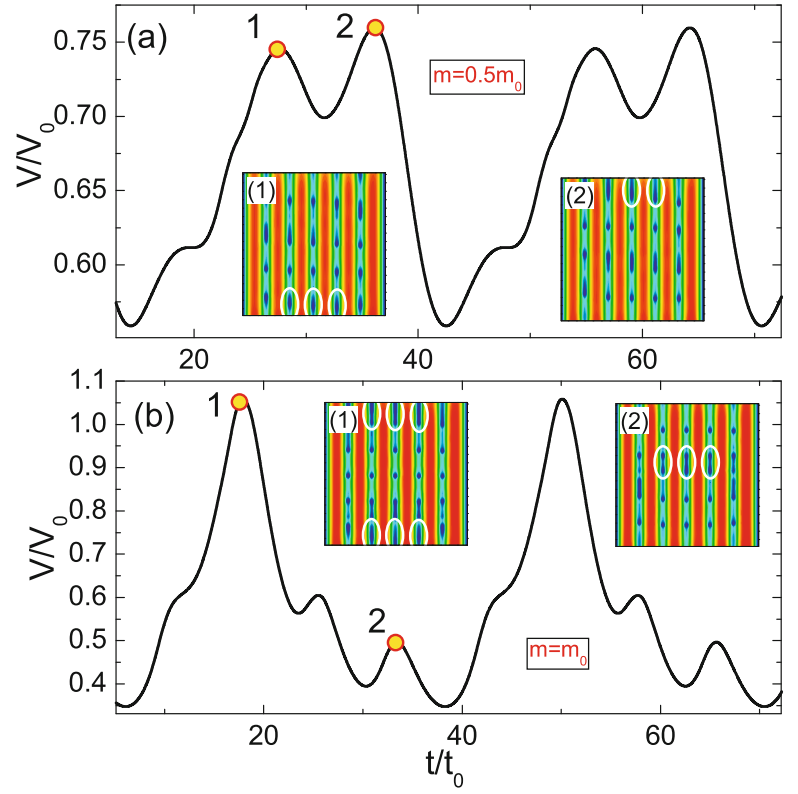

Fig. 5. The same as in Figure 4 but for $j=0.16 j_{0}$.

though an external AC forcing. As an example, we consider sinusoidal AC current with a static DC offset, so that the total current is given by:

$$
j=j_{d c}+\left|j_{a c}\right| \sin \left(2 \pi t \nu_{0}\right)
$$

where $\nu$ is the frequency of the current oscillations. We restrict ourselves to small DC currents and a weak AC signal to avoid current-induced vortex generation.

As a main result, we show in Figure 6 the Fourier power spectrum $\left(\tilde{V}(\nu)=1 / \sqrt{2 \pi} \int V(t) e^{i 2 \pi t \nu} d t\right)$ of the voltage vs. time curves obtained in the presence of $\mathrm{DC}$ (Fig. 6a) $\left(j_{d c}=0.08 j_{0}\right)$ and $\mathrm{AC}$ (Figs. 6b-6d) $(j=$ $\left.0.08 j_{0}+0.01 \sin \left(2 \pi t \nu_{0}\right)\right)$ currents for different values of the frequency of the applied drive $\nu_{0}$. The applied magnetic field is $H=0.08 H_{0}$ and the magnetization of the dots is set at $M=0.5 M_{0}$. In the absence of an AC current (Fig. 6a), the dynamics of the system is characterized by a characteristic frequency $\nu^{*}=0.0074 t_{0}^{-1}$, which is determined by the crossing of a vortex across the system. The few higher frequency peaks in the spectrum are attributed to the hopping of vortices from one magnetic dot to the other. When an AC component is added to a DC current, extra peaks appear in the spectrum, which correspond to the frequency of the applied drive (Figs. 6b and $6 \mathrm{c}$ ). A well-separated signal at the characteristic frequency is still present. The additional peaks are clearly visible at frequencies of the applied drive larger than $\nu^{*}$ (Fig. 6d). At these frequencies, the vortices in all junctions move in phase (i.e., the superradiant state), and the motion of fluxons is synchronized with the frequency of the AC current.

Figure 7 shows the results obtained for the magnetization of the $\operatorname{dots} M=M_{0}$, applied DC current $j=0.08 j_{0}$ and magnetic field $H=0.08 H_{c 2}$. For this set of external parameters, the resistive state is also characterized by the generation of vortex-antivortex pairs (see Fig. 4b).

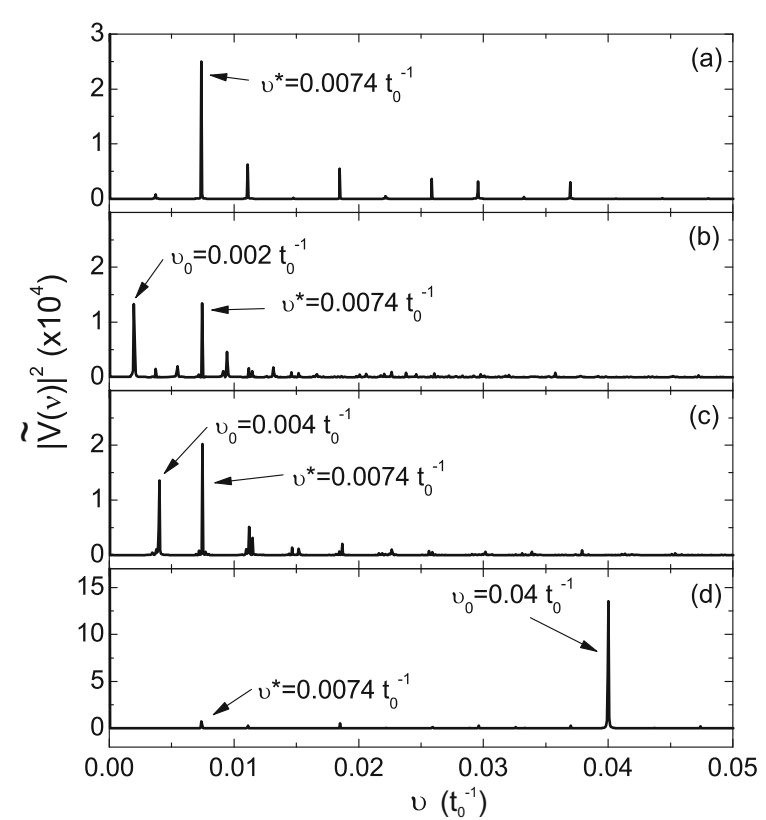

Fig. 6. Fourier power spectrum $|\tilde{V}(\nu)|^{2}$ of the voltage curves obtained in the presence of $\mathrm{DC}$ (a) $\left(j_{d c}=0.08 j_{0}\right)$ and $\mathrm{AC}$ (b) $-(\mathrm{d})\left(j=0.08 j_{0}+0.01 \sin \left(2 \pi t \nu_{0}\right)\right)$ currents. The frequency of the applied drive $\nu_{0}$ is given in the figures. The applied magnetic field is $H=0.08 H_{0}$ and the magnetization of the dots is $M=0.5 M_{0} . \nu^{*}$ is the characteristic frequency of the system determined by the crossing of a vortex across the system.

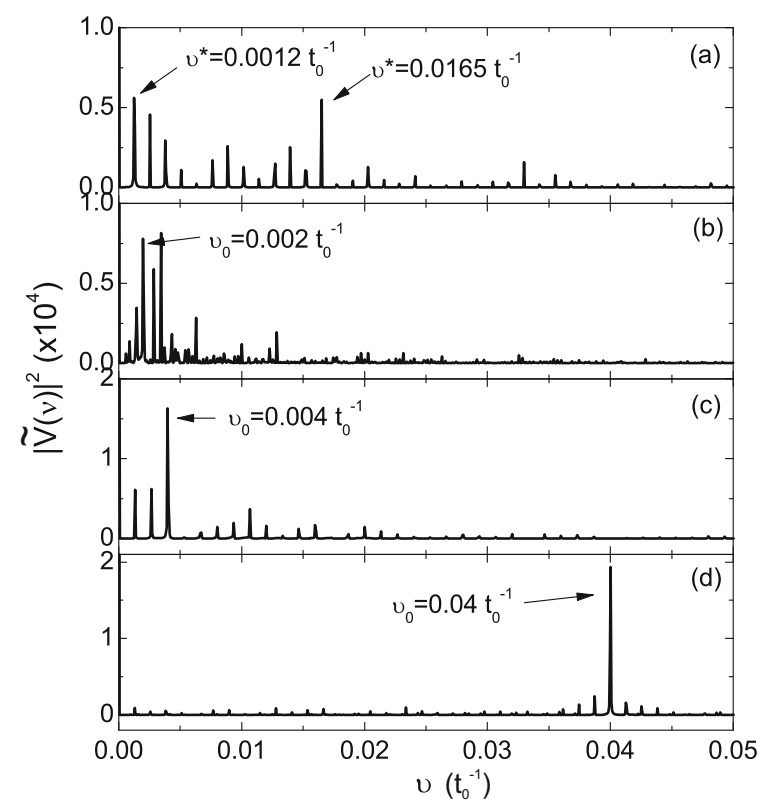

Fig. 7. The same as in Figure 6 but for $M=M_{0}$.

Many well-separated peaks are observed in the spectrum (see Fig. 7a). Among them, one can identify two characteristic frequencies $\nu^{*}=0.0012 t_{0}^{-1}$ and $\nu^{*}=0.0165 t_{0}^{-1}$, corresponding to the crossing of fluxons across the sample and the vortex hopping from one pinning to the other, respectively. Interestingly, both of these signals disappear from the spectrum in the presence of low frequency $\mathrm{AC}$ 
drive (see Fig. 7b). Additional small background peaks are obtained in the spectrum, indicating chaotic motion of vortices in the system. With increasing frequency of the $\mathrm{AC}$ current, their motion becomes more ordered resulting in well separated peaks corresponding to resonance frequencies (Figs. 7c and 7d). We find only peaks corresponding to the frequency of the external drive, which indicates a modulation of the Josephson oscillations by the AC drive. However, the amplitude of the peaks in the Fourier spectra is smaller as compared to the case when magnetic dots with smaller magnetization are present (compare Figs. 6d and $7 \mathrm{~d}$ ). Thus, the synchronization of the motion of Josephson vortices using applied $A C$ drive becomes difficult for larger magnetization of the magnetic dots due to formation of vortex-antivortex pairs. The same qualitative behavior of Josephson vortices is observed for magnetic dots with different size and periodicity, as well as for different parameters of the Josephson junctions.

\section{Conclusions}

Using the anisotropic time-dependent Ginzburg-Landau theory we investigate the dynamics of Josephson vortices in artificial stacks of superconducting-normalsuperconducting Josephson junctions in the presence of a periodic pinning potential created by out-of-plane magnetized magnetic dots. Depending on the value of the applied current, we have identified two states for the moving vortex lattice: i) an out-of-phase motion at small currents (e.g., a triangular lattice) and ii) a coherent superradiant state at larger currents. We also studied the possibility of synchronizing the motion of vortices using an external AC drive. We found that the superradiant state can be realized for small dot magnetization, i.e., weak pinning potential. Synchronization becomes difficult for larger magnetization of the dots due to the formation of vortex-antivortex pairs. Our findings can be useful for developing high-frequency emission devices, where the tunability of the Josephson oscillations is required.

This work was supported by EU Marie Curie (Project No. 253057).

\section{References}

1. T. Koyama, M. Tachiki, Solid State Commun. 96, 367 (1995)

2. Yu.I. Latyshev, M.B. Gaifullin, T. Yamashita, M. Machida, Y. Matsuda, Phys. Rev. Lett. 87, 247007 (2001)

3. S. Ooi, T. Mochiku, K. Hirata, Phys. Rev. Lett. 89, 247002 (2002)

4. M. Machida, Phys. Rev. Lett. 90, 037001 (2003)

5. A.V. Ustinov, N.F. Pedersen, Phys. Rev. B 72, 052502 (2005)

6. S. Savel'ev, V. Yampol'skii, A. Rakhmanov, F. Nori, Phys. Rev. B 72, 144515 (2005)

7. M. Tachiki, M. Iizuka, K. Minami, S. Tejima, H. Nakamura, Phys. Rev. B 71, 134515 (2005)
8. L.N. Bulaevskii, A.E. Koshelev, Phys. Rev. Lett. 97, $267001(2006)$

9. M.-H. Bae, H.-J. Lee, J.-H. Choi, Phys. Rev. Lett. 98, $027002(2007)$

10. S.O. Katterwe, V.M. Krasnov, Phys. Rev. B 80, 020502 (2009)

11. F. Marchesoni, S. Savel'ev, M. Tachiki, F. Nori, Phys. Rev. B 81, 174531 (2010)

12. G.R. Berdiyorov, S.E. Savel'ev, M.V. Milosevic, F.V. Kusmartsev, F.M. Peeters, Phys. Rev. B 87, 184510 (2013)

13. G.R. Berdiyorov, S.E. Savel'ev, F.V. Kusmartsev, F.M. Peeters, Supercond. Sci. Technol. 26, 125010 (2013)

14. X. Hu, S.-Z. Lin, Supercond. Sci. Technol. 23, 053001 (2010)

15. S. Savel'ev, V.A. Yampol'skii, A.L. Rakhmanov, F. Nori, Rep. Prog. Phys. 73, 026501 (2010)

16. A.K. Jain, K.K. Likharev, J.E. Lukens, J.E. Sauvageau, Phys. Rep. 109, 309 (1984)

17. S. Madsen, N.F. Pedersen, Phys. Rev. B 72, 134523 (2005)

18. I. Martin, G.B. Halász, L.N. Bulaevskii, A.E. Koshelev, J. Appl. Phys. 108, 033908 (2010)

19. G. Costabile, R. Monaco, S. Pagano, G. Rotoli, Phys. Rev. B 42, 2651 (1990)

20. N.F. Pedersen, A. Davidson, Phys. Rev. B 41, 178 (1990)

21. N. Grønbech-Jensen, Phys. Rev. B 47, 5504 (1993)

22. N. Grønbech-Jensen, M. Cirillo, Phys. Rev. B 50, 12851 (1994)

23. S. Madsen, N. Grønbech-Jensen, Phys. Rev. B 71, 132506 (2005)

24. A.E. Koshelev, I.S. Aranson. Phys. Rev. Lett. 85, 3938 (2000)

25. A.V. Chiginev, V.V. Kurin, Phys. Rev. B 70, 214523 (2004)

26. Y. Yamada, K. Nakajima, T. Yasuda, T. Yamashita, K. Nakajima, IEEE Trans. Appl. Supercond. 15, 1028 (2005)

27. Y. Yamada, K. Nakajima, K. Nakajima, Physica C 470, 1130 (2010)

28. P. Spathis, M. Konczykowski, C.J. van der Beek, P. Gierlowski, M. Li, P.H. Kes, Phys. Rev. B 76, 104518 (2007)

29. A.E. Koshelev, Yu. I. Latyshev, M. Konczykowski, Phys. Rev. B 74, 104509 (2006)

30. S. Savel'ev, F. Nori, Nat. Mater. 1, 179 (2002)

31. Y.-D. Jin, G.-H. Lee, H.-J. Lee, J. Supercond. Nov. Magn. 23, 1071 (2010)

32. G.R. Berdiyorov, M.V. Milošević, F.M. Peeters, Phys. Rev. B 80, 214509 (2009)

33. M.V. Milošević, G.R. Berdiyorov, F.M. Peeters, Phys. Rev. Lett. 95, 147004 (2005)

34. M.V. Milošević, G.R. Berdiyorov, F.M. Peeters, Phys. Rev. B 75, 052502 (2007)

35. G.R. Berdiyorov, M.M. Doria, A.R. de C. Romaguera, M.V. Milošević, E.H. Brandt, F.M. Peeters, Phys. Rev. B 87, 184508 (2013)

36. L.R. Motowidlo, B.A. Zeitlin, M.S. Walker, P. Haldar, Appl. Phys. Lett. 61, 991 (1992)

37. L. Kramer, R.J. Watts-Tobin, Phys. Rev. Lett. 40, 1041 (1978)

38. R.J. Watts-Tobin, Y. Krähenbühl, L. Kramer, J. Low Temp. Phys. 42, 459 (1981)

39. G.R. Berdiyorov, A.R. de C. Romaguera, M.V. Milošević, M.M. Doria, L. Covaci, F.M. Peeters, Eur. Phys. J. B 85, 130 (2012) 
40. M.L. Latimer, G.R. Berdiyorov, Z.L. Xiao, W.K. Kwok, F.M. Peeters, Phys. Rev. B 85, 012505 (2012)

41. G.R. Berdiyorov, X.H. Chao, F.M. Peeters, H.B. Wang, V.V. Moshchalkov, B.Y. Zhu, Phys. Rev. B 86, 224504 (2012)

42. G. Berdiyorov, K. Harrabi, J.P. Maneval, F.M. Peeters, Supercond. Sci. Technol. 28, 025004 (2015)

43. C.Y. Liu, G.R. Berdiyorov, M.V. Milošević, Phys. Rev. B 83, 104524 (2011)

44. A.I. Gubin, K.S. Il'in, S.A. Vitusevich, M. Siegel, N. Klein, Phys. Rev. B 72, 064503 (2005)

45. R. Kato, Y. Enomoto, S. Maekawa, Phys. Rev. B 47, 8016 (1993)

46. T.Y. Hsiang, J. Clarke, Phys. Rev. B 21, 945 (1980)

47. G.R. Berdiyorov, A.D. Hernandez, F.M. Peeters, Phys. Rev. Lett. 103, 267002 (2009)

48. G.R. Berdiyorov, A.K. Elmurodov, F.M. Peeters, D.Y. Vodolazov, Phys. Rev. B 79, 174506 (2009)
49. G.R. Berdiyorov, M.V. Milošević, L. Covaci, F.M. Peeters, Phys. Rev. Lett. 107, 177008 (2011)

50. A. Andronov, I. Gordion, V. Kurin, I. Nefedov, I. Shereshevsky, Physica C 213, 193 (1993)

51. G.R. Berdiyorov, M.V. Milošević, F.M. Peeters, Phys. Rev. B 79, 184506 (2009)

52. A.V. Silhanek, M.V.M.V. Milošević, R.B.G. Kramer, G.R. Berdiyorov, J. Van de Vondel, R.F. Luccas, T. Puig, F.M. Peeters, V.V. Moshchalkov, Phys. Rev. Lett. 104, 017001 (2010)

Open Access This is an open access article distributed under the terms of the Creative Commons Attribution License (http://creativecommons.org/licenses/by/4.0), which permits unrestricted use, distribution, and reproduction in any medium, provided the original work is properly cited. 